\title{
Effects of Early Enteral Glutamine Supplementation on Intestinal Permeability in Critically Ill Patients
}

\author{
Zahra Vahdat Shariatpanahi ${ }^{1}$, Ghazaleh Eslamian ${ }^{2}$, Seyed Hossein Ardehali ${ }^{3}$, Ahmad-Reza Baghestani $^{4}$
}

\begin{abstract}
Background and aims: Enteral administration of glutamine has been proposed as an effective recovery of intestinal barrier function. This amino acid has a modulating effect on the reducing bacterial translocation, which can influence immune functions of the intestine. The objective was to evaluate the effects of early enteral glutamine supplementation on intestinal permeability in critically ill patients.

Materials and methods: A total of 80 critically ill patients older than 18 years were randomly assigned to one of two groups according to the stratified blocked randomization by age and admission category. Consecutive participants took enteral formula plus $0.3 \mathrm{~g} / \mathrm{kg} / \mathrm{day}$ glutamine powder or enteral formula plus maltodextrin during the ICU stay for a maximum of 10 days. Plasma glutamine, endotoxin, zonulin, and antiendotoxin immunoglobulin (lg) G/lgM concentrations were measured on days 5 and 10 of intervention.

Results: Out of 80 participants, 36 patients in the glutamine group and 34 patients in the control group were included in the analysis of the outcomes. Enteral glutamine significantly reduced plasma zonulin concentration up to $40 \%$ during 10 days. This reduction was significantly greater compared with that of the placebo group $(P<0.001)$. Endotoxin concentration decreased in both groups; this reduction was significantly greater in the glutamine group ( $p=0.014)$. The antiendotoxin IgM and IgG antibody levels increased in the glutamine group but decreased in the control group $(p<0.001)$. There were no significant differences in clinical outcomes between two groups.

Conclusion: Early enteral glutamine supplementation led to a declined intestinal permeability in critically ill patients.

Keywords: Antiendotoxin immunoglobulin, Endotoxin, Enteral nutrition, Glutamine, Zonulin

Indian Journal of Critical Care Medicine (2019): 10.5005/jp-journals-10071-23218
\end{abstract}

\section{INTRODUCTION}

The altered gut function is a common problem in the critically ill patients. ${ }^{1,2}$ Delayed gastric emptying, abnormal motility patterns, and impaired intestinal barrier integrity are commonly reported in the intensive care unit (ICU). ${ }^{1}$ The leaky gut may play an important role in the development of catabolic states in critically ill patients. ${ }^{3}$ It has been proposed that bacterial translocation and gut-origin sepsis may be involved in the pathogenesis of systemic infectious complications and multiple organ deficiency syndromes. ${ }^{4}$ Conditions leading to intestinal hypoperfusion such as hemorrhagic shock, trauma, burns, pancreatitis, and major abdominal operations cause breakdown of the intestinal mucosal barrier. ${ }^{3}$

Previous studies have illustrated a link between glutamine supplementation and preservation of enteric mucosa integrity. ${ }^{5}$ Glutamine is the preferred metabolic fuel for enterocytes and plays a key role in maintaining intestinal barrier integrity and function. ${ }^{6}$ Although glutamine is classified as a nonessential amino acid, it may become conditionally indispensable in hypermetabolic states. ${ }^{7}$ Glutamine is reduced in critically ill patients. ${ }^{8}$ Experimental studies determined that depletion of glutamine can be viewed as a compromised body defense system, which leads to villus atrophy, decreased expression of tight junction proteins in several conditions, and increased intestinal permeability. ${ }^{6}$ Glutamine has a modulating effect on the reducing bacterial translocation, which can influence immune functions of the intestine. ${ }^{8}$ Intestinal permeability is most commonly measured by fractional urinary excretion of orally ingested probes, circulating biomarkers of gut barrier function, and circulating zonulin level, the only physiological modulator of intercellular tight junctions. ${ }^{9}$ Assessing the effects of enteral glutamine on intestinal permeability in critically ill and burned patients shows diverse results. ${ }^{10-14}$

\begin{abstract}
${ }^{1}$ Department of Clinical Nutrition and Dietetics, Faculty of Nutrition and Food Technology, National Nutrition and Food Technology Research Institute, Shahid Beheshti University of Medical Sciences, Tehran, Iran ${ }^{2}$ Student Research Committee, Department of Clinical Nutrition and Dietetics, Faculty of Nutrition and Food Technology, National Nutrition and Food Technology Research Institute, Shahid Beheshti University of Medical Sciences, Tehran, Iran

${ }^{3}$ Department of Anesthesiology and Critical Care, Shohadaye Tajrish Hospital, Shahid Beheshti University of Medical Sciences, Tehran, Iran

${ }^{4}$ Department of Biostatistics, Physiotherapy Research Center, Faculty of Paramedical Sciences, Shahid Beheshti University of Medical Sciences, Tehran, Iran
\end{abstract}

Corresponding Author: Ghazaleh Eslamian, Student Research Committee, Department of Clinical Nutrition and Dietetics, Faculty of Nutrition and Food Technology, National Nutrition and Food Technology Research Institute, Shahid Beheshti University of Medical Sciences, Tehran, Iran, e-mail: gh_eslamian@yahoo.com

How to cite this article: Shariatpanahi ZV, Eslamian G, Ardehali SH, Baghestani AR. Effects of Early Enteral Glutamine Supplementation on Intestinal Permeability in Critically III Patients. Indian J Crit Care Med 2019;23(8):356-362.

Source of support: Nil

Conflict of interest: None

Today, supplementation of glutamine is both a confusing and controversial topic. According to the last revision of American Society for Parenteral and Enteral Nutrition guideline, enteral glutamine exerts a trophic effect in maintaining gut integrity. However, supplemental enteral glutamine is not recommended to be added to an enteral nutrition (EN) regimen routinely in critically ill patients. ${ }^{15}$

() The Author(s). 2019 Open Access This article is distributed under the terms of the Creative Commons Attribution 4.0 International License (https://creativecommons. org/licenses/by-nc/4.0/), which permits unrestricted use, distribution, and non-commercial reproduction in any medium, provided you give appropriate credit to the original author(s) and the source, provide a link to the Creative Commons license, and indicate if changes were made. The Creative Commons Public Domain Dedication waiver (http://creativecommons.org/publicdomain/zero/1.0/) applies to the data made available in this article, unless otherwise stated. 
We hypothesize that after enteral glutamine administration, plasma antiendotoxin immunoglobulin $\mathrm{G}(\mathrm{lg} \mathrm{G})$ and $\operatorname{lgM}$ would increase while plasma zonulin and endotoxin levels decrease as the biomarkers of intestinal permeability. Therefore, to evaluate this hypothesis, a clinical trial was established to determine effects of early enteral glutamine supplementation on intestinal permeability in ICU-hospitalized patients.

\section{Materials and Methods}

\section{Study Design and Participants}

The design of the current study was a randomized, double-blind, placebo-controlled trial. In this study, 80 critically ill patients were recruited between April and October 2017 from general ICU of a university hospital. After a full review of the inclusion and exclusion criteria and explanation of the risks and benefits of the study, critically ill patients meeting the inclusion criteria of the study were enrolled. The study was approved by the related ethics committee and performed in accordance with the ethical standards laid down in the 1964 Declaration of Helsinki and its later amendments. The funder of the current study had no role in its design or conduct; in the collection, management, analysis, or interpretation of the data; or in the preparation, review, or approval of the manuscript.

Consecutive adult patients ( $>18$ years old) were screened for eligibility according to the following inclusion criteria: Start of study intervention within $48 \mathrm{~h}$ after ICU admission; having the EN for at least $72 \mathrm{~h}$ aiming for full EN and receiving at least $80 \%$ of enteral formula during the first $48 \mathrm{~h}$; and submitting written informed consent by the patients or their legal representative. On the other hand, the patients were excluded for one or more of the following criteria: $>24 \mathrm{~h}$ from admission to the ICU; requiring other specific EN for medical reason such as renal or liver failure; death or discharge before day 5; any absolute contraindication to receive EN; pregnancy or lactating; body mass index less than $18 \mathrm{~kg} / \mathrm{m}^{2}$ or greater than $40 \mathrm{~kg} / \mathrm{m}^{2}$; not being expected to be in ICU for more than $48 \mathrm{~h}$ due to imminent death; liver cirrhosis -Child's class C liver disease; seizure disorder requiring anticonvulsant; history of allergy or intolerance to the study product components; receiving glutamine within two weeks before starting the study; and enrollment or planned enrollment in a related ICU interventional study.

Subject participation in the study was prematurely discontinued in the following situations: further participation was a health risk for the patient; a subject or legal representative who gave informed consent decided to resign from further participation in the study; and a patient might require a hospital transfer.

\section{Randomization, Masking, and Interventions}

Figure 1 presents the details about the recruitment, randomization, and follow-up of the current study. Randomization was stratified by age (18-65 and $\geq 65$ years) and admission category (medical, surgical, and trauma). Patients that meet all the inclusion criteria and none of the exclusion criteria were assigned using computergenerated randomization to receive 10 days of enteral formula plus $0.3 \mathrm{~g} / \mathrm{kg} /$ day glutamine powder or isocaloric enteral formula plus maltodextrin. ENTERA Meal formula (54.6\% carbohydrate, $14 \%$ protein, and $31.6 \%$ fat), maltodextrin, and glutamine were provided by Karen Pharma \& Food Supplement, Co., Tehran, Iran. The group assignments were concealed in sealed envelopes and opened at enrollment by somebody who was blinded to all baseline assessments.

\section{Administration of Intervention}

Study product administration was started within $48 \mathrm{~h}$ after admission at the ICU and ended at discharge from the ICU or at end of the study (day 10). Planned enteral tube feeding (nasogastric or gastrostomy tube feeding) was administered after stabilizing the hemodynamic condition of each patient. Administration of formula was done by the intermittent method every four hours. The volume of administered tube feed was determined based on the calorie needs and tolerance of patients by starting from $50 \mathrm{~mL}$. Through the first $24 \mathrm{~h}$, if the patient could tolerate the feeding, the volume of administration was increased to the goal volume. The supplements were administrated three times a day. General recommended energy and protein intakes for critically ill patients -based on weight and metabolic condition- were 25-30 $\mathrm{kcal} / \mathrm{kg}$ and $1.2-2 \mathrm{~g} / \mathrm{kg}$, respectively. ${ }^{15}$ According to the protein calculation, if a patient had required an extra amount of protein, modular protein supplements would have been added to the enteral formula. The volume and calorie intake were recorded on a daily basis. The volume ratio (VR) was calculated to determine the efficacy of daily nutritional administration: VR $(\%)=$ (volume received/volume prescribed) $\times 100$.

\section{Procedures}

Baseline measurements were performed before starting EN with study product on day 1 . At the screening visit, age, sex, length, medical history, preexisting conditions, medication use, enteral route, feeding regimen, date of hospital and ICU admission, and diagnosis for ICU admission were recorded. Medication and nutritional supplements used from two weeks before start study product until discharge from the ICU or the end of the study were also recorded. At baseline, the Acute Physiology and Chronic Health Evaluation (APACHE II), ${ }^{16}$ Sequential Organ Failure Assessment (SOFA) score, ${ }^{17}$ and Nutrition Risk score in the Critically III (NUTRIC) ${ }^{18}$ were determined over the first $24 \mathrm{~h}$ after admission at the ICU. From the start of feeding study product until day 10 , the adverse events, gastrointestinal intolerance, the net amount of tube feeding intake, and mortality were assessed on a daily basis.

Venous blood samples were taken at baseline within $2 \mathrm{~h}$ before the start of intervention and between 7 am and 9 am on day 5 and Day 10 for laboratory data. Blood samples were collected in EDTAcontaining tubes and plasma was separated by centrifugation at $3,000 \mathrm{rpm}$ for $10 \mathrm{~min}$ at $4^{\circ} \mathrm{C}$. Plasma samples were snap-frozen and stored in small aliquots at $-80^{\circ} \mathrm{C}$ to prevent repeated freezing and thawing until the determination of laboratory data.

\section{Primary and Secondary Outcomes}

Plasma endotoxin, zonulin, and antiendotoxin IgG and IgM concentrations were the primary outcome measures. Endotoxin concentrations in plasma were measured by a commercially available quantitative chromogenic endpoint Limulus Amebocyte Lysate (LAL) QCL-1000 kit (Lonza, Walkersville, MD). ${ }^{19}$ Enzyme-linked immunosorbent assay (ELISA, Zonulin ELISA Kit, Immundiagnostik, Bensheim, Germany) was used to determine plasma zonulin concentration. Plasma antiendotoxin core antibody IgG and IgM concentrations were determined by an enzyme-linked immunosorbent assay (Chromogenix, Quadritech Diagnostics, Epsom, UK). All assays were performed according to manufacturers' manual; tests were carried out in duplicates. The glutamine concentration in plasma was measured using the high-performance liquid chromatography, as described previously. ${ }^{20}$ 


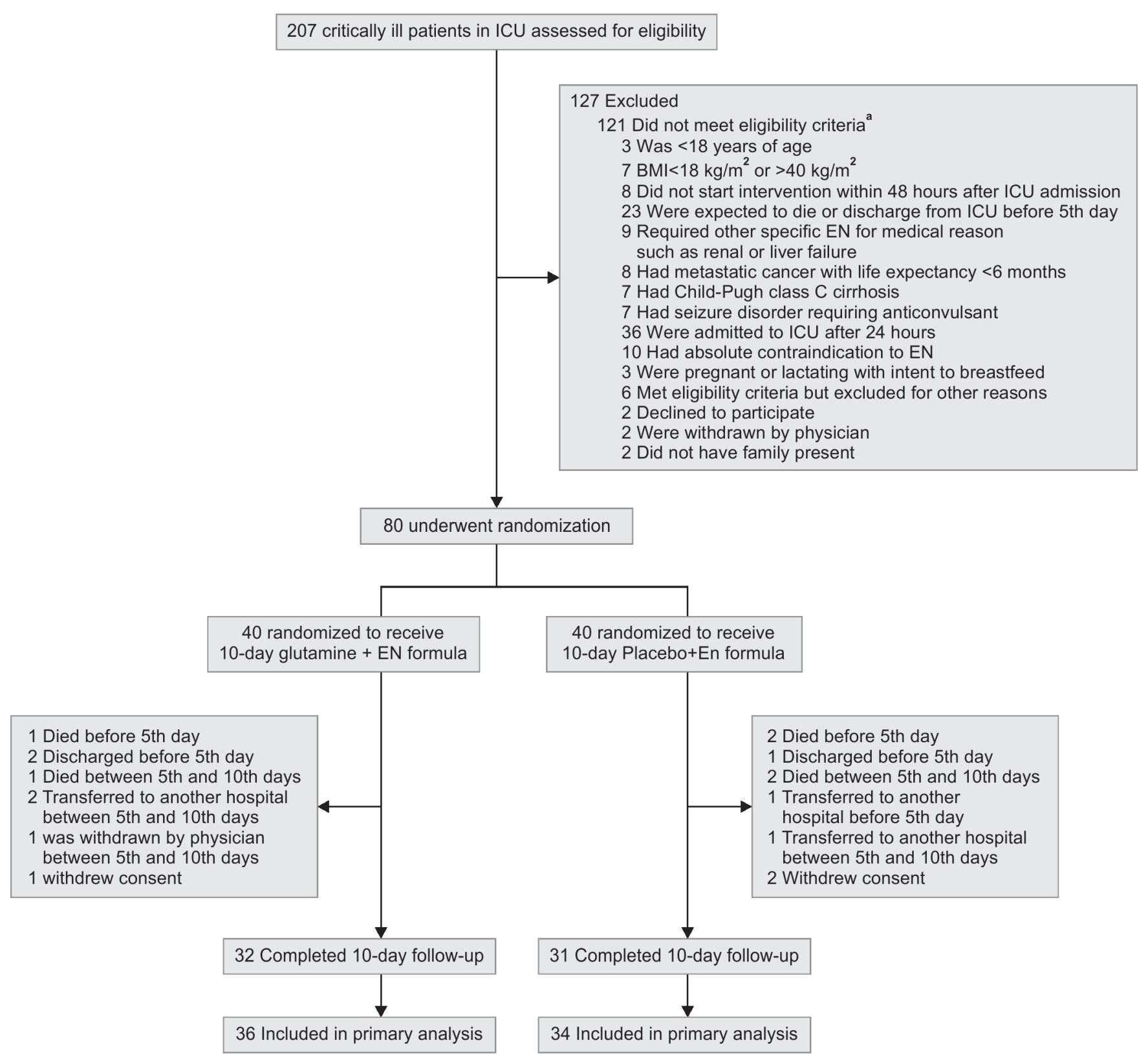

Fig. 1: Study enrollment and randomization of critically ill patients

Secondary outcomes included incidence of sepsis according to the American College of Chest Physicians and the Society of Critical Care Medicine, ${ }^{21}$ mortality at ICU, length of stay (LOS) in ICU, and incidence of gastrointestinal complications such as abdominal distention, vomiting, diarrhea, and constipation.

\section{Statistical Analysis}

Data were analyzed by applying the SPSS software (version 20; Chicago, IL, USA). All hypothesis tests were two-tailed, with $\mathrm{P}<0.05$ denoting the statistical significance. Data were presented as frequencies and percentages, means and $95 \%$ confidence intervals (Cls) or standard deviations, or medians and interquartile ranges (IQR), when appropriate. Chi-square or Fisher exact tests were used to evaluate the differences in the distribution of categorical variables, and Mann-Whitney test was used to check the differences in the distribution of continuous variables between intervention and control, as most of the variables were highly skewed and not normally distributed. The data were analyzed according to the intention-to-treat (ITT) principle. Participants missing laboratory measurements of the primary and secondary outcome measures were imputed using the regression method. Means and 95\% $\mathrm{Cls}$ for changes from baseline in primary outcomes were compared at day 5 and day 10 using the ANCOVA.

\section{Results}

\section{Recruitment and Follow-up}

Patient screening, enrollment, and retention by treatment groups are shown in Figure 1. Out of 80 participants, 32 patients in the glutamine group (80\%) and 31 patients in the control group (77.8\%) completed the 10-day follow-up of the study. Baseline characteristics of participants completing the study were similar to 
Table 1: Baseline characteristics of study patients

\begin{tabular}{|c|c|c|c|}
\hline Characteristic & $\begin{array}{l}\text { Glutamine group } \\
n=36\end{array}$ & $\begin{array}{l}\text { Control group } \\
n=34\end{array}$ & p value \\
\hline Age, yr, median (IQR) & $62(53-70)$ & $64(53-70)$ & $0.728^{*}$ \\
\hline Sex, No. (\%) & & & $0.782^{* *}$ \\
\hline Male & $20(55.6)$ & $20(58.8)$ & \\
\hline Female & $16(44.4)$ & $14(41.2)$ & \\
\hline Admission category, No (\%) & & & $0.967^{* *}$ \\
\hline Medical & $17(47.2)$ & $15(44.1)$ & \\
\hline Surgical & $12(33.3)$ & $12(35.3)$ & \\
\hline Trauma & $7(19.5)$ & $7(20.6)$ & \\
\hline \multicolumn{4}{|l|}{ Admision scores, median(IQR) } \\
\hline APACHE-II & $24(19-47)$ & $24(19-37)$ & $0.888^{*}$ \\
\hline NUTRIC & $5(4-6)$ & $5(4-6)$ & $0.485^{*}$ \\
\hline SOFA & $6(5-7)$ & $6(5-7)$ & $0.779^{*}$ \\
\hline \multicolumn{4}{|c|}{ Baseline blood levels, median(IQR) } \\
\hline Albumin, g/dL & $2.7(1.6-3.3)$ & $2.5(1.7-4.4)$ & $0.612^{*}$ \\
\hline Glutamine, mg/dL & $373(309-411)$ & $387(298-466)$ & $0.879^{*}$ \\
\hline $\begin{array}{l}{ }^{*} \text { Mann-Whitney test } \\
{ }^{* *} \mathrm{X}^{2} \text { test or Fisher exact test } \\
\text { APACHE-II, Acute Physiology } \\
\text { interquartile range; NUTRIC, } \\
\text { Sequential Organ Failure Asse }\end{array}$ & col & - & $x_{1}$ \\
\hline
\end{tabular}

Table 2: Feeding details of study patients

\begin{tabular}{llll}
\hline & Glutamine & Control & \\
Enteral feeding administration & $n=36$ & $n=34$ & $p$ value \\
\hline Time from ICU admission to study EN start, median (IQR), hr & $28(18-41)$ & $30(19-41)$ & $0.510^{*}$ \\
Duration of administration, median (IQR), d & $10(8-10)$ & $10(7-10)$ & $0.808^{*}$ \\
Mean planned energy intake, median (IQR), kcal/d & $1497(1447-1584)$ & $1506(1408-1594)$ & $0.809^{*}$ \\
Mean planned protein intake, median (IQR), g/d & $73(65-83)$ & $72(66-80)$ & $0.426^{*}$ \\
Mean energy intake, median (IQR), kcal/d & $1375(1218-1483)$ & $1352(1240-1486)$ & $0.261^{*}$ \\
Mean protein intake, median (IQR), g/d & $64(57-80)$ & $61(56-71)$ & $0.101^{*}$ \\
VR at day 1, median (IQR), \% & $86(83-92)$ & $85(83-90)$ & $0.545^{*}$ \\
VR at day 5, median (IQR), \% & $89(86-94)$ & $88(85-91)$ & $0.219^{*}$ \\
VR at day 10, median (IQR), \% & $90(83-96)$ & $86(82-94)$ & $0.173^{*}$ \\
Patients with supplemental parenteral nutrition, No (\%) & $5(13 \%)$ & $4(11 \%)$ & $0.537^{* *}$ \\
\hline
\end{tabular}

${ }^{*}$ Mann-Whitney test

${ }^{* *} X^{2}$ test

$E N$, enteral nutrition; ICU, intensive care unit; IQR, interquartile range; VR, volume ratio

those who did not complete the protocol of the study. There were 70 patients in the final ITT analysis.

\section{Baseline Characteristics of the Patients}

Baseline demographic and medical characteristics are presented in Table 1. The study participants had a mean age of 59 years and $57 \%$ of them were male. As a result of the stratified randomization design, the age and admission category distribution were similar in the both groups.

The nutritional data of patients in both groups during the intervention are shown in Table 2. No statistically significant differences were observed between study groups in mean duration of study product administration, the time of starting nutritional support, the energy intake, the protein intake, or total volume of study product administered.

The baseline median (IQR) plasma glutamine of participants was $483(418-516)$ and $494(409-577) \mu \mathrm{mol} / \mathrm{mL}$ in the glutamine and control groups, respectively, demonstrating no significant difference ( $p=0.503)$. At the end of the 10-day treatment period, median [IQR] plasma glutamine levels were significantly higher in the glutamine group compared with the control patients (588 $\mu \mathrm{mol} / \mathrm{mL}[462-707 \mu \mathrm{mol} / \mathrm{mL}$ ] versus $472 \mu \mathrm{mol} / \mathrm{mL}$ [440-536 $\mu \mathrm{mol} /$ $\mathrm{mL}], \mathrm{P}=0.006$ ). No adverse events were attributed to glutamine supplementation.

\section{Primary Outcome}

The baseline plasma levels of intestinal permeability markers of the two groups were similar $(P<0.05)$. Table 3 presents the mean changes of plasma levels of intestinal permeability markers. Plasma endotoxin concentration decreased in both groups on days 5 and 10; however, the reduction in the glutamine group was significantly greater than that in the placebo group. The change in the endotoxin concentration on days 5 and 10 showed a significant difference between those treated with glutamine or placebo. Furthermore, 


\begin{tabular}{|c|c|c|c|}
\hline Change from baseline & $\begin{array}{l}\text { Glutamine } \\
n=36\end{array}$ & $\begin{array}{l}\text { Control } \\
n=34\end{array}$ & pvalue* \\
\hline \multicolumn{4}{|c|}{ Plasma endotoxin concentration (EU/mL) } \\
\hline Day 5 & $-0.26(-0.36,-0.16)$ & $-0.09(-0.25,0.07)$ & 0.002 \\
\hline Day 10 & $-0.40(-0.52,-0.28)$ & $-0.24(-4.69,-0.01)$ & 0.014 \\
\hline \multicolumn{4}{|c|}{ Plasma zonulin concentration ( $\mathrm{ng} / \mathrm{mL}$ ) } \\
\hline Day 5 & $-1.13(-1.59,-0.67)$ & $0.39(0.06,0.71)$ & $<0.001$ \\
\hline Day 10 & $-2.06(-2.78,-1.33)$ & $0.85(0.17,1.53)$ & $<0.001$ \\
\hline \multicolumn{4}{|c|}{ Plasma antiendotoxin $\lg G(\mathrm{MU} / \mathrm{mL})$} \\
\hline Day 5 & $22.58(13.27,31.90)$ & $-7.21(-16.29,1.88)$ & $<0.001$ \\
\hline Day 10 & $52.03(32.39,71.67)$ & $-29.79(-39.96,-19.63)$ & $<0.001$ \\
\hline \multicolumn{4}{|c|}{ Plasma antiendotoxin IgM (MU/mL) } \\
\hline Day 5 & $4.14(-0.98,9.26)$ & $-7.91(-13.70,-2.13)$ & 0.005 \\
\hline Day 10 & $15.44(6.61,24.28)$ & $-16.71(-25.45,-7.96)$ & $<0.001$ \\
\hline
\end{tabular}

"Based on an ANCOVA model that regressed changes from baseline on treatment group, baseline value of the outcome, albumin and energy intake

Table 4: Comparison of gastrointestinal complication diagnosed after initiation of intervention between groups

\begin{tabular}{llll}
\hline & Glutamine & Control & \\
Gastrointestinal complication, No (\%) & $n=36$ & $n=34$ & pvalue \\
\hline Abdominal distention & $11(30)$ & $11(32)$ & 0.538 \\
Vomiting & $3(8)$ & $3(9)$ & 0.635 \\
Diarrhea & $3(8)$ & $9(26)$ & 0.044 \\
Constipation & $6(17)$ & $13(38)$ & 0.039 \\
\hline
\end{tabular}

${ }^{*} \mathrm{X}^{2}$ test, Fisher axact test

glutamine supplementation produced a significant decrease in the plasma zonulin concentration on days 5 and 10. Plasma levels of IgG and IgM antibodies increased in the glutamine group significantly on days 5 and 10 compared with the placebo group. However, at the end of the 10-day treatment period, a significant decrease in IgG and IgM antibodies levels was seen in the control group.

\section{Secondary Outcomes}

The results obtained from the analysis of gastrointestinal complication are presented in Table 4. As can be noted, there was no significant difference in terms of the incidence of abdominal distention and vomiting between both groups. The incidence of diarrhea was significantly lower in the glutamine group (8\%) than in the control group (26\%). The incidence of constipation was also significantly lower in the glutamine group (17\%) than in the control group (38\%).

There were no statistically significant differences in the incidence of new severe sepsis between groups. Overall, $5.6 \%$ of those in the glutamine group $(95 \% \mathrm{Cl} ; 0-13.9 \%)$ versus $11.8 \%$ in the control group $(95 \% \mathrm{Cl}, 2.8-23.5 \% ; p=0.309)$ had new severe sepsis. There were no statistically significant differences for mortality at ICU between the glutamine group (19\%, 95\% Cl; 8-33\%) and the control group (12\%, 95\%Cl; 3-24\%; $p=0.378)$. The median (IQR) LOS in ICU was 10 (8-20) days and 13 (7-29) days for the glutamine group and the control group, respectively $(p=0.646)$.

\section{Discussion}

The current study provided valuable information on whether the administration of enteral glutamine could alter intestinal permeability in critically ill patients. Assessing the plasma endotoxin, zonulin, and antiendotoxin IgG and IgM concentrations in this study showed that intestinal permeability was improved after a short period of glutamine-supplemented enteral feedings. In addition, glutamine supplementation was associated with a significant decrease in the incidence of diarrhea and constipation. No effect of glutamine was observed on any other gastrointestinal complications, mortality, and LOS in ICU. Both groups were comparable in terms of baseline characteristics. Most patients tolerated EN and achieved the planned caloric goal within the first 2 days of treatment.

Glutamine as a major fuel source for both enterocytes and colonocytes plays a critical role in activating the mammalian target of rapamycin cell signaling in enterocytes. Therefore, it is necessary for the maintenance of intestinal structure in both normal and stressed states. ${ }^{22}$ During critical illness, nutrient deficiencies can further be exacerbated probably due to the impaired immune function and a higher incidence of developing infectious complications, multiorgan failure, and death. Lower plasma and skeletal muscle glutamine levels contribute to immune dysfunction, indicating elevated glutamine needs in critically ill patients. ${ }^{23}$ Glutamine may augment immune system response by lowering the incidence of bacteria and toxin translocation from the intestinal lumen to the systemic circulation. ${ }^{24}$ The effects of glutamine on intestinal permeability and its role in maintaining functional integrity of the gut have been investigated in humans and animal models. ${ }^{25}$ In most previous clinical trials in critical care, urinary lactulose and mannitol ratio (L/M) was determined as a gut permeability marker. ${ }^{10,12-14}$ Dual sugar absorption tests have some technical limitations. These limitations are related to timing of specimen acquisition, handling of urine, and assay performance. ${ }^{26}$ Some studies have evaluated the plasma endotoxin level as a marker of intestinal permeability with enteral glutamine supplementation in severe burn patients. ${ }^{11,12}$ In the present study, we were able to demonstrate the significant positive effects of enteral glutamine supplementation on intestinal permeability by determining plasma endotoxin, zonulin, and antiendotoxin immunoglobulin IgG and IgM concentrations. Endotoxin is the major component of the outer membrane of gram-negative bacteria. Breakdown of the intestinal mucosal barrier resulted in the passage of endotoxin into the systemic circulation. ${ }^{27}$ In our study, the plasma endotoxin level was markedly decreased after 
using enteral glutamine for 10 days, compared with the control group. Our result is in line with studies of Peng et al. ${ }^{12}$ and Zhou et al., ${ }^{13}$ which were conducted in severe burned patients after using enteral glutamine. According to the present study, glutamine supplementation was able to reduce plasma zonulin level. Zonulin as a master regulator of intercellular tight gap junctions is the only measurable physiological blood protein that reflects the intestinal permeability. Moreover, the increased zonulin levels are considered to be a marker of the impairment of intestinal barrier function. ${ }^{28}$ A recent study has shown that critically ill patients have elevated serum levels of zonulin-1. ${ }^{29}$ Klaus et al. demonstrated the elevated levels of plasma zonulin in septic patients. ${ }^{30}$ To our knowledge, zonulin is not considered as an outcome in previous clinical trials in critical care. Our results suggest that the zonulin assay is a reasonable choice to use as a marker for intestinal permeability in ICU-hospitalized patients. In this regard, L/M-ratio test provides challenging results according to the time of urine collection and fasting time, especially when considering that the participants in our study were the critically ill patients. Glutamine can promote plasma antiendotoxin IgG and IgM by effectively improving the immune system of patients. Consistent with our results, Wang et al. showed that after glutamine-enriched nutritional support in patients with advanced gastric cancer during perioperative chemotherapy, plasma IgG and IgM levels were significantly higher in the study group compared to the control group. ${ }^{31}$

In our study, there was also no discernible difference in the secondary outcomes relating to ICU-acquired sepsis, mortality, and LOS in ICU. These results support previous meta-analysis studies $^{32-34}$ including the REDOX ${ }^{35}$ and METAPLUS ${ }^{36}$ trials indicating that glutamine supplementation given to a mixed population of critically ill patients does not significantly affect ICU mortality and LOS in ICU. In contrast, early systematic reviews have shown that glutamine supplementation may be associated with a reduction in complication and mortality rates and LOS in ICU in trauma, burn, and mixed ICU patients. ${ }^{37,38}$ Previous clinical studies have demonstrated that low plasma glutamine values $(<420 \mu \mathrm{mol} / \mathrm{L})$ upon admission are related to increased mortality. ${ }^{39}$ In our study, mean baseline glutamine was $488 \mu \mathrm{mol} / \mathrm{L}$ and $27 \%$ of patients had glutamine values smaller than $420 \mu \mathrm{mol} / \mathrm{L}$.

The majority of critically ill patients have at least one gastrointestinal symptom during their ICU stay. ${ }^{40}$ The reduced incidence of mucositis, diarrhea, and neuropathy with glutamine supplementation among patients under chemotherapy has been evidenced in recent studies. ${ }^{41}$ Our results showed that enteral glutamine supplementation decreases the occurrences of diarrhea, which is consistent with the result of a prospective randomized trial of enteral glutamine in critical illness. ${ }^{42}$ It suggests that the beneficial impact of glutamine supplementation on diarrhea may contribute to gastrointestinal mucosa rather than the host immune response. ${ }^{43}$ Our results showed that dietary enteral glutamine supplementation may decrease the occurrences of constipation. The mechanism of glutamine effect on constipation is thought to involve regulated endogenous gut microbiota, based upon an animal study. ${ }^{44}$

The most important strength of the current study was its design as a randomized, double-blind, placebo-controlled trial. The study also has some limitations. Body weight was recorded based on recent patient file information of subject's or relative's recall. The group of critically ill patients is very diverse, which makes it difficult to select a homogenous population with minimal variation in outcome parameters. The duration of ICU stay of patients also varies widely because some patients were tube-fed for only few days and others for more than a month. Another limitation is that the study patients were not followed up further to assess the sustainability of the results obtained after the termination of intervention.

\section{Conclusion}

In conclusion, this study found some evidence that a glutaminesupplemented enteral diet in critically ill patients can decrease intestinal permeability and gastrointestinal complications. Despite this clear benefit, we found no effect on mortality at ICU and duration of stay in ICU. These findings should be confirmed in future randomized, placebo-controlled studies, which also include a washout period and larger sample sizes to analyze the subtypes of admission category in critically ill patients with glutamine deficiency.

\section{References}

1. Hill LT. Gut dysfunction in the critically ill- mechanisms and clinical implications. Southern African Journal of Critical Care. 2013;29(1):1115.

2. De-Souza DA, Greene LJ. Intestinal permeability and systemic infections in critically ill patients: effect of glutamine. Crit Care Med. 2005;33(5):1125-1135.

3. Sertaridou E, Papaioannou V, Kolios G, Pneumatikos I. Gut failure in critical care: old school versus new school. Ann Gastroenterol. 2015;28(3):309-322.

4. Deitch EA. Gut-Origin sepsis; evolution of a concept. Surgeon. 2012;10(6):350-356.

5. Savy GK. Glutamine supplementation. Heal the gut, help the patient. J Infus Nurs. 2002;25(1):65-69.

6. Achamrah N, Dechelotte P, Coeffier M. Glutamine and the regulation of intestinal permeability: from bench to bedside. Curr Opin Clin Nutr Metab Care. 2017;20(1):86-91.

7. Coeffier M, Dechelotte P. The role of glutamine in intensive care unit patients: mechanisms of action and clinical outcome. Nutr Rev. 2005;63(2):65-69.

8. Labow BI, Souba WW. Glutamine. World J Surg. 2000;24(12):1503-1513.

9. Galipeau HJ, Verdu EF. The complex task of measuring intestinal permeability in basic and clinical science. Neurogastroenterol Moti. 2016;28(7):957-965.

10. Uranjek J, Vovk I, Kompan L. Effect of the route of glutamine supplementation (enteral versus parenteral) on intestinal permeability on surgical intensive care unit patients: a pilot study. Surgical Practice. 2013;17(4):153-160.

11. Conejero R, Bonet A, Grau T, Esteban A, Mesejo A, Montejo JC, et al. Effect of a glutamine-enriched enteral diet on intestinal permeability and infectious morbidity at 28 days in critically ill patients with systemic inflammatory response syndrome: a randomized, singleblind, prospective, multicenter study. Nutrition. 2002;18(9):716-721.

12. Peng X, Yan H, You Z, Wang P, Wang S. Effects of enteral supplementation with glutamine granules on intestinal mucosal barrier function in severe burned patients. Burns. 2004;30(2):135-139.

13. Zhou YP, Jiang ZM, Sun YH, Wang XR, Ma EL, Wilmore D. The effect of supplemental enteral glutamine on plasma levels, gut function, and outcome in severe burns: a randomized, double-blind, controlled clinical trial. JPEN J Parenter Enteral Nutr. 2003;27(4):241-245.

14. Velasco N, Hernandez G, Wainstein C, Castillo L, Maiz A, Lopez F, et al. Influence of polymeric enteral nutrition supplemented with different doses of glutamine on gut permeability in critically ill patients. Nutrition. 2001;17(11-12):907-911. 
15. McClave SA, Taylor BE, Martindale RG, Warren MM, Johnson DR, Braunschweig $C$, et al. Guidelines for the Provision and Assessment of Nutrition Support Therapy in the Adult Critically III Patient: Society of Critical Care Medicine (SCCM) and American Society for Parenteral and Enteral Nutrition (ASPEN). JPEN J Parenter Enteral Nutr. 2016;40(2):159-211.

16. Knaus WA, Draper EA, Wagner DP, Zimmerman JE. APACHE II: a severity of disease classification system. Crit Care Med. 1985;13(10):818-829.

17. Vincent JL, Moreno R, Takala J, Willatts S, De Mendonca A, Bruining $\mathrm{H}$, et al. The SOFA (Sepsis-related Organ Failure Assessment) score to describe organ dysfunction/failure. On behalf of the Working Group on Sepsis-Related Problems of the European Society of Intensive Care Medicine. Intensive Care Med. 1996;22(7):707-710.

18. Heyland DK, Dhaliwal R, Jiang X, Day AG. Identifying critically ill patients who benefit the most from nutrition therapy: the development and initial validation of a novel risk assessment tool. Crit Care. 2011;15(6):R268.

19. Harmon P, Cabral-Lilly D, Reed RA, Maurio FP, Franklin JC, Janoff A. The release and detection of endotoxin from liposomes. Anal Biochem. 1997;250(2):139-146.

20. van Eijk HM, Wijnands KA, Bessems BA, Olde Damink SW, Dejong $\mathrm{CH}$, Poeze M. High sensitivity measurement of amino acid isotope enrichment using liquid chromatography-mass spectrometry. J Chromatogr B Analyt Technol Biomed Life Sci. 2012;905:31-36.

21. American College of Chest Physicians/Society of Critical Care Medicine Consensus Conference: definitions for sepsis and organ failure and guidelines for the use of innovative therapies in sepsis. Crit Care Med. 1992;20(6):864-874.

22. Wang B, Wu G, Zhou Z, Dai Z, Sun Y, Ji Y, et al. Glutamine and intestinal barrier function. Amino acids. 2015;47(10):2143-2154.

23. Calder PC, Yaqoob P. Glutamine and the immune system. Amino acids. 1999;17(3):227-241.

24. Swank GM, Deitch EA. Role of the gut in multiple organ failure: bacterial translocation and permeability changes. World J Surg. 1996;20(4):411-417.

25. Kim MH, Kim H. The Roles of Glutamine in the Intestine and Its Implication in Intestinal Diseases. Int J Mol Sci. 2017;18(5).

26. Dorshow RB, Hall-Moore C, Shaikh N, Talcott MR, Faubion WA, Rogers $\mathrm{TE}$, et al. Measurement of gut permeability using fluorescent tracer agent technology. Sci Rep. 2017;7.

27. Rietschel ET, Kirikae T, Schade FU, Mamat U, Schmidt G, Loppnow $\mathrm{H}$, et al. Bacterial endotoxin: molecular relationships of structure to activity and function. FASEB J. 1994;8(2):217-225.

28. Fasano A. Zonulin, regulation of tight junctions, and autoimmune diseases. Ann N Y Acad Sci. 2012;1258:25-33.

29. Greis C, Rasuly Z, Janosi RA, Kordelas L, Beelen DW, Liebregts $\mathrm{T}$. Intestinal T lymphocyte homing is associated with gastric emptying and epithelial barrier function in critically ill: a prospective observational study. Crit Care. 2017;21(1):70.
30. Klaus DA, Motal MC, Burger-Klepp U, Marschalek C, Schmidt EM, Lebherz-Eichinger $D$, et al. Increased plasma zonulin in patients with sepsis. Biochem Med. 2013;23(1):107-111.

31. Wang J, Li Y, Qi Y. Effect of glutamine-enriched nutritional support on intestinal mucosal barrier function, MMP-2, MMP-9 and immune function in patients with advanced gastric cancer during perioperative chemotherapy. Oncol Lett. 2017;14(3):3606-3610.

32. Oldani M, Sandini M, Nespoli L, Coppola S, Bernasconi DP, Gianotti L. Glutamine Supplementation in Intensive Care Patients: A MetaAnalysis of Randomized Clinical Trials. Medicine. 2015;94(31):e1319.

33. Chen QH, Yang Y, He HL, Xie JF, Cai SX, Liu AR, et al. The effect of glutamine therapy on outcomes in critically ill patients: a metaanalysis of randomized controlled trials. Crit Care. 2014;18(1):R8.

34. van Zanten AR, Dhaliwal R, Garrel D, Heyland DK. Enteral glutamine supplementation in critically ill patients: a systematic review and meta-analysis. Crit Care. 2015;19:294.

35. Heyland D, Muscedere J, Wischmeyer PE, Cook D, Jones G, Albert M, et al. A randomized trial of glutamine and antioxidants in critically ill patients. N Engl J Med. 2013;368(16):1489-1497.

36. van Zanten AR, Sztark F, Kaisers UX, Zielmann S, Felbinger TW, Sablotzki AR, et al. High-protein enteral nutrition enriched with immune-modulating nutrients vs standard high-protein enteral nutrition and nosocomial infections in the ICU: a randomized clinical trial. JAMA. 2014;312(5):514-524.

37. Novak F, Heyland DK, Avenell A, Drover JW, Su X. Glutamine supplementation in serious illness: a systematic review of the evidence. Crit Care Med. 2002;30(9):2022-2029.

38. Avenell A. Glutamine in critical care: current evidence from systematic reviews. Proc Nutr Soc. 2006;65(3):236-241.

39. Oudemans-van Straaten HM, Bosman RJ, Treskes M, van der Spoel $\mathrm{HJ}$, Zandstra DF. Plasma glutamine depletion and patient outcome in acute ICU admissions. Intensive Care Med. 2001;27(1):84-90.

40. Reintam A, Parm P, Kitus R, Kern H, Starkopf J. Gastrointestinal symptoms in intensive care patients. Acta Anaesthesiol Scand. 2009;53(3):318-324.

41. Jolfaie NR, Mirzaie S, Ghiasvand R, Askari G, Miraghajani M. The effect of glutamine intake on complications of colorectal and colon cancer treatment: A systematic review. J Res Med Sci. 2015;20(9):910-918.

42. Hall JC, Dobb G, Hall J, de Sousa R, Brennan L, McCauley R. A prospective randomized trial of enteral glutamine in critical illness. Intensive Care Med. 2003;29(10):1710-1716.

43. Yalcin SS, Yurdakok K, Tezcan I, Oner L. Effect of glutamine supplementation on diarrhea, interleukin-8 and secretory immunoglobulin A in children with acute diarrhea. J Pediatr Gastroenterol Nutr. 2004;38(5):494-501.

44. Zhang Y, Lu T, Han L, Zhao L, Niu Y, Chen H. L-Glutamine Supplementation Alleviates Constipation during Late Gestation of Mini Sows by Modifying the Microbiota Composition in Feces. BioMed Research International. 2017;2017. 